\title{
SOME FEATURES OF TRANSVERSE INSTABILITY OF PARTLY COMPENSATED PROTON BEAMS
}

\author{
Vadim Dudnikov \\ Fermi National Accelerator Laboratory*, Batavia, IL 60510, USA
}

\begin{abstract}
Transverse e-p instability in the proton rings could be damped by increase a beam density and the rate of secondary particles generation above a threshold level, corresponded a decrease of the unstable wavelength below a transverse beam size. In high current proton rings as LANSCE PSR is possible to reach this "Island of stability" by fast, concentrated charge exchange injection without painting and enhanced generation of secondary plasma as it was demonstrated in small scale PSR at BINP [1]. After damping of e-p instability has been accumulated a circulating proton beam with intensity, corresponding a tune shift $\Delta v=0.85 \times 6$ in the ring with $v=0.85$. Transverse instability of a bunched beam was damped by a simple feed back [2,3]. Experimental observations of a transverse instability of proton beams in different accelerators and storage rings will be discussed. The methods of instability damping will be considered. These experimental dates could be useful for verification of computer simulation tools developed for investigation of space charge and instabilities in realistic conditions $[4,5]$.
\end{abstract}

\section{EXPERIMENTAL OBSERVATION OF E-P INSTABILITIES}

Limitation of beam intensity and performance by repulsing space charge effects is accepted in general as a "natural" limit and a reaching of this limit is often a design goal of the projects in physics and technology. Some time this limit could be over passed with use of space charge neutralization (compensation) by particles with an opposite charge. Development of the industrial scale electromagnetic isotope separation in the Manhattan Project is an example of very successful development of the space charge neutralization $(\mathrm{SCN})$ of heavy ion beam with increase a beam intensity from microamps to hundreds of milliamps. Now this SCN is a basis for ion implantation in the Semiconductor industry. But SCN is very delicate and nonequilibrium process and fast development of the strong instability can destroy neutralization and beam propagation. Often any admixture of compensating particles can drive the beam instability with a low threshold intensity, far below a space charge limit. A circulating beam in the accelerators and storage rings is more sensitive to any influences, then the beams with finite transportation. An increase of beam intensity and a brightness is increase a nonequilibrium, and instability driving by compensating particles can start in every part of accelerator complex from ion source to the

Work supported by the U.S. Department of Energy under contract No. DE-AC02-76CH03000. Dudnikov@,fnal.gov booster. A special cures should be used to avoid the instability. The importance of the transverse beam instability driving by interaction with a plasma compensating particles has been considered in the first proposals of the high intense beam production as the Stabilized relativistic beam [6]. In the analyzes of stability of partly compensated electron beam by Chirikov [7] has been shown that threshold intensity and level of compensation for instability of coasting circulating beam can be low. In this model of electron-ion instability low energy compensating particles (electrons or ions) are trapped within the space- charge potential of the circulating beam. Coupled transverse oscillations of the beam and the trapped particles has develop with a transformation of the energy of beam to the oscillation energy, leading to the beam loss. For prevention of the beam degradation by compensating particles in electronpositron colliders from the first projects were used clearing electrodes for remove secondary particles along all orbits, and with a very deep vacuum this was enough for avoiding this instability.

A transverse instability of the bunched proton beam driven by compensating particles has been observed at 1965 in small scale storage ring used for development of charge exchange injection [2,3]. In this small PSR with circumference $\mathrm{L}=2.5 \mathrm{~m}$ by charge exchange injection of 1 $\mathrm{MeV} \mathrm{H}^{-}$during 800 turns was reached linear proton density $\lambda \sim 2.510^{9} \mathrm{p} / \mathrm{cm}$, a volume density $n \sim 10^{8} \mathrm{~cm}^{-3}$ and corresponding beam potential relative the chamber wall $\mathrm{U} \sim 1 \mathrm{kV}$. This instability with a fast loss of bunched beam has been stabilized by simple negative feed back with pick up electrode, resonance amplifier and deflection electrode. At 1967 in other small PSR was observes an instability of coasting proton beam with a low threshold, connected with an accumulation of the compensating electrons $[1,8]$. The behavior of this instability was in good agreement with a Chirikov's analyses [7]. This instability has been damped by increase of the bounce frequency of electrons in the proton beam with increase of positive ion density. A stable circulating proton beam with completely compensated space charge and with intensity, corresponding a tune shift $\Delta v=0.85 \times 6$ in the ring with $v=0.85$ (up to 9 time above a space charge limit) was accumulated $[1,8]$. These results were discussed in the reviews $[10,11]$.

Observation of transverse instabilities in the AGS and in the ZGS has been presented in the Cambridge Accelerator Conference 1967 along with [8] but only recent was identified as e-p instability. Instability of the coasting 
proton beam with accumulation of electrons have been observed in the Bevatron and CERN ISR at 1971. In the Bevatron this instability was damped by feed back and by beam bunching, and in ISR by improving a vacuum from $10^{-10}$ Torr to $10^{-11}$ Torr and increase a number of clearing electrodes. A similar instability with accumulation of positive ions in negative charged electron and antiproton beams, has been observed in synchrotron radiation source ALADDIN and in the CERN and Fermilab antiproton accumulators [12]. Instability in AA was dumped by using a sophisticated methods if ion repulsing as beam shaking in supplement to vacuum improving and clearing by electric field.

Further theoretical analyses of this instability of dipole and quadruple oscillation has been developed at 1972 by Koshkarev and Zenkevich [13] and has been extended in

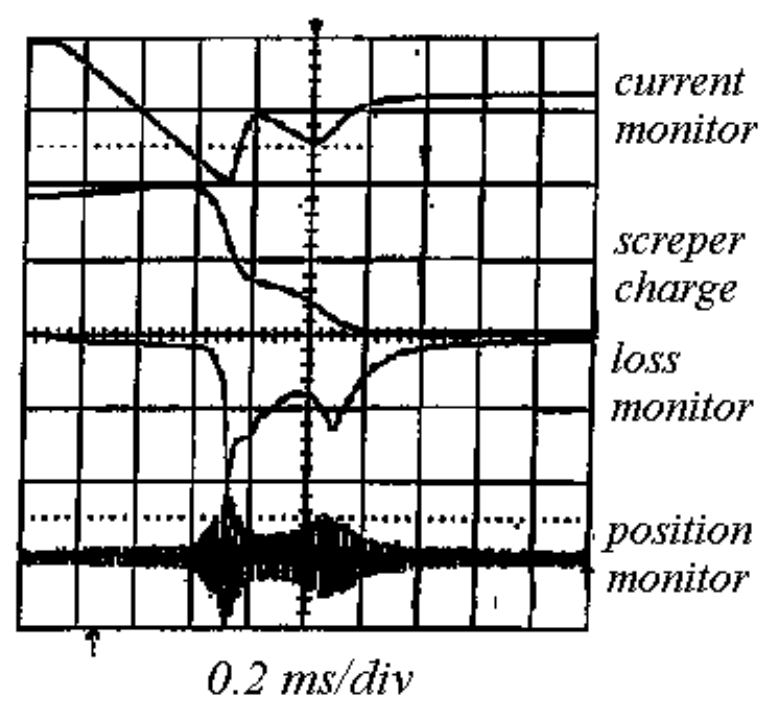

Figure 1: Oscillograms of coasting beam instability in the LANSCE PSR

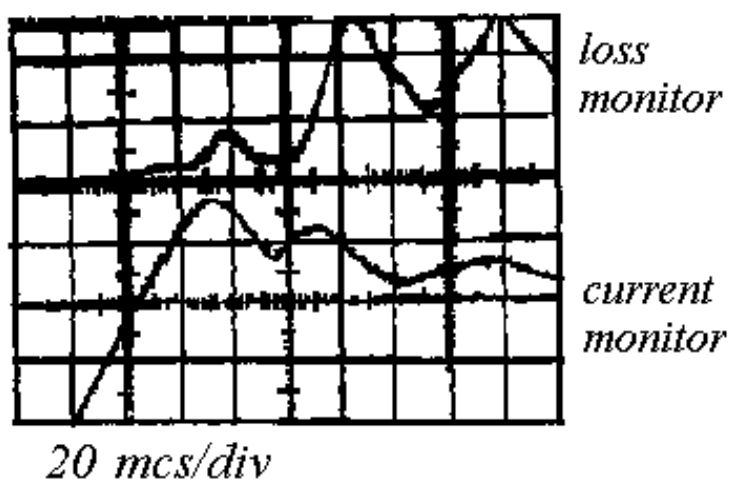

Figure 2: Coasting beam instability in the INP PSR.

other publications.

New attention to the e-p instability was attracted after observation in the LANSE proton storage ring (PSR) a strong transverse instability with loss of bunched and unbunched beam [14]. This unpredicted and "mysterious" instability during 20 years limit the pulsed intensity of spallation neutron source in the level, below design goal.
After many investigation was reached a good understanding of coasting beam instability and good agreement with theoretical models, but instability of the bunched beam have not an acceptable understanding. Typical development of an e-p instability of coasting beam in the LANSCE PSR shown in Fig.1. It is absolutely identical to the corresponding picture from the INP PSR in Fig.2. In several workshops have been discussed different aspects of the e-p instability in different accelerators and storage rings and further development for understanding and damping of the instability has been considered [15-17]. Transverse instability of coasting beam was observed at the BNL Booster and in Fermilab Booster with a DC field.

With an increase of beam density the bounce frequency of secondary particles oscillations is increase and significant development of oscillation could be reached during one pass of bunch or trine of bunch through the portion of secondary particles accumulated during only one pass of the bunch or bunch trine. The attention to this possibility " Fast beam- ion instability" has been attracted in paper [18]. This type of instability has been observed in the ALS with an increased He gas density [19]. But this " Fast beam- ion instability" has been observed in low energy negative ion beam after ion source at 1975 [1] and after FNAL $0.75 \mathrm{MeV}$ preaccelerator [20]. Space charge compensation by ions exhibit essential differences in comparison with compensation by electron one and corresponding instability have some features connected with mass difference and the ability to keep coherence.

\section{DAMPING OF E-P INSTABILITY}

For low beam density an electron bounce frequency $\omega^{2}=4 c^{2} r_{e} n_{i}$ is comparable with a revolution frequency and bounce oscillation coupled with a low modes of betatron oscillations. For low modes, a magnitude of electron oscillations is more larger the beam oscillations and electrons removing from the beam by very small oscillations of protons. This mechanism used for remove ions from the antiproton beam. For a strong instability is need to have a high beam density and a strong enough source of a secondary particles. Suppression of a secondary particles generation and accumulation is a "traditional" method for e-p instability suppression: improve vacuum, use a gap for electron removing, use a cleaning electrodes, suppressing a secondary emission. A feed back system could be efficient in instability damping. Progress in suppressing of e-p instability in LA PSR was discussed in reports of R. Macek, presented in [15-17,21]. The need for higher beam intensity at PSR and for future high-intensity, proton drivers has motivated a multi-lab collaboration (LANL, ANL, FNAL, LBNL, BNL, ORNL, PPPL) to undertake research to better understand causes, dynamics and cures for e-p instability. Important characteristics of the electron cloud were recently measured with ANL electron analyzers and various collection electrodes [22]. Suppression of e generation by TiN coatings has confirmed the importance of secondary emission processes in generating the electron cloud. New 
tests of potential controls included dual harmonic RF, damping by higher order multipoles, damping by $\mathrm{X}, \mathrm{Y}$ coupling and the use of inductive inserts to compensate longitudinal space charge forces. Use of a skew quadrupole, heated inductive inserts and higher RF voltage has enabled the PSR to accumulate stable beam intensity up to $9.7 \mathrm{mC}\left(610^{13} \mathrm{p} / \mathrm{p}\right)$, which is a significant increase $(60 \%)$ over the previous maximum of $6 \mathrm{mC}$. This beam was stable with a high rate of secondary electron production.

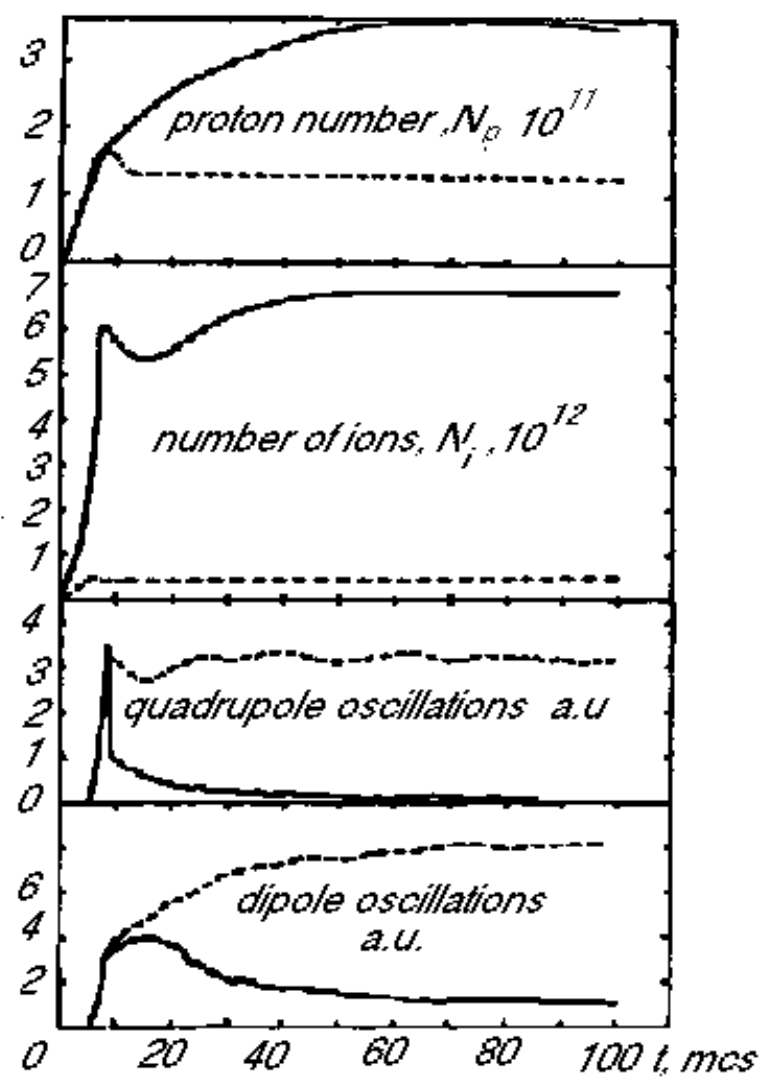

Figure 3: Accumulation of circulating protons in the INP PSR before critical intensity (dotted lines) and above a critical intensity (solid lines) with damping of instabilities and overcoming a space charge limit.

Efficient damping of a e-p instability by increase a beam current density and increase a rate of secondary plasma generation has been demonstrated in small scale PSR at INP, Novosibirsk, at $1976[1,8]$. This process of the proton accumulation by charge- exchange injection is shown in Fig.3. With injected current below $2 \mathrm{~mA}$ and low gas density observed a fast excitation of dipole and quadrupole coherent oscillations and proton accumulation is saturated by loss on the level of $\mathrm{N}_{\mathrm{p}}=1.210^{11}$ as shown by dotted lines. With increase an injected current and gas density above a threshold level the accumulation dynamic changed dramatically, as shown by solid lines. A number of circulating proton increase during the all injection time and limited only by injected current. Oscillations are damped after a short, fast growing. The density of secondary ion and electron in the beam increase dramatically. This increase of positive ion density up to $n=3-410^{8} \mathrm{~cm}^{-3}$ have shift the bounce frequency of electron oscillation out of the bend of instability. Nonlinear, anomaly fast accumulation of secondary plasma is important for e-p stabilization. With injection current of $5 \mathrm{~mA}, 1 \mathrm{MeV} \mathrm{H}^{-}$up to $1.810^{12}$ protons has been accumulated in PSR with a perimeter of $6 \mathrm{~m}$. This intensity is corresponded a tune shift of $\Delta v=0.85 \times 6$ in the PSR with $v=0.85$. This increase of beam and ion density above a second threshold for e-p instability could be used for production of extremely bright ion beams with increase a brightness by nonliowille charge exchange injection and for acceleration of high current ion beams in recirculators with an inductance linacs. It is looks as space charge compensation of the bunched beam is also possible.

\section{REFERENCES}

[1] G.Budker, G.Dimov, and V.Dudnikov, et al., Proc.X Internat. Conf. Particle Accelerators, Protvino, 1977, v.2,p.287, 1978.

[2] G.Budker, G. Dimov, and V. Dudnikov, Proc. Internat. Symp. Electron and Positron Storage Ring, France, Sakley,1966, rep. VIII, 6.1 (1966)

[3] G. Budker, G. Dimov, and V. Dudnikov, Soviet Atomic Energy, 22, 384 (1967).

[4] M. Reiser, The physics of High Brightness Beam, p.112, World Sci. 1999.

[5] R. Davidson et al., in [17].

[6] G. Budker, Soviet Atomic Energy, 5, 9 (1956).

[7] B. Chirikov, Soviet Atomic Energy, 19, 239 (1965).

[8] G.Budker, G.Dimov, V. Dudnikov, V. Shamovsky,

Proc. sixth Internat. Conf. on High Energy Accelerators, 1967, MIT\&HU,A-104, CEAL-2000, 1967, p.422.

[9] G.Dimov, V. Chupriyanov, Particle accelerators, 14, 155-84 (1984).

[10] V. Dudnikov, A. Shabalin, HIPAC'92, v.2,1025, Hamburg, 1993.

[11] V. Dudnikov, in [17].

[12] J. Marriner, Yu. Orlov, et.al., Particle Accelerators, 30, 13 (1990).

[13] D. Koshkarev and P. Zenkevich, Part. Accel., 3, 1-9 (1972).

[14] E. Colton, D. Nuffer, G. Swain, et al., Particle Accelerators, 23,133 (1988).

[15] Proc.of the Workshop on Accelerators for Future Spallation Neutron Sources, 1993, Santa Fe, NM.LA-UR-93-1356, Vol. II A,p.412-449(1993).

[16] Proceedings of the Santa Fe Workshop on electron Effect, 1997, LA-UR-98-1601.

[17] ICFA Mini- Workshop on Two- Stream Instabilities in Particle Accelerators and Storage Rings, Santa Fe, 2000 (www.aps.anl.gov/conferences/icfa/two-stream). [18] G.Stupakov, T. Raubenheimer and F. Zimmermann, Phys. Rev. E 52 (5), 5499 (1995).

[19] J.Byrd et al., Phys. Rev. Let. 79(1),79 (1997).

[20] E. McCrory et al., Linac 88, CEBAF-89-001, p.182(1989). M. Popovic, T. Sullivan, Linac2000.

[21] R. Macek, in [15-17] and this conference.

[22] A. Browman, in [17]. 